\title{
An improved computational method in structural dynamics
}

\begin{abstract}
Purpose: In structural, earthquake and aeronautical engineering and mechanical vibration, the solution of dynamic equations for a structure subjected to dynamic loading leads to a high order system of differential equations. The numerical methods are usually used for integration when either there is dealing with discrete data or there is no analytical solution for the equations. Since the numerical methods with more accuracy and stability give more accurate results in structural responses, there is a need to improve the existing methods or develop new ones. The paper aims to discuss these issues. Design/methodology/approach: In this paper, a new time integration method is proposed mathematically and numerically, which is accordingly applied to single-degree-of-freedom (SDOF) and multi-degree-of-freedom (MDOF) systems. Finally, the results are compared to the existing methods such as Newmark's method and closed form solution. Findings: It is concluded that, in the proposed method, the data variance of each set of structural responses such as displacement, velocity, or acceleration in different time steps is less than those in Newmark's method, and the proposed method is more accurate and stable than Newmark's method and is capable of analyzing the structure at fewer numbers of iteration or computation cycles, hence less timeconsuming. Originality/value: A new mathematical and numerical time integration method is proposed for the computation of structural responses with higher accuracy and stability, lower data variance, and fewer numbers of iterations for computational cycles.
\end{abstract}

Keyword: Computational method; Direct time integration methods; Newmark's method; Parviz' method; Structural dynamics; Structural response 